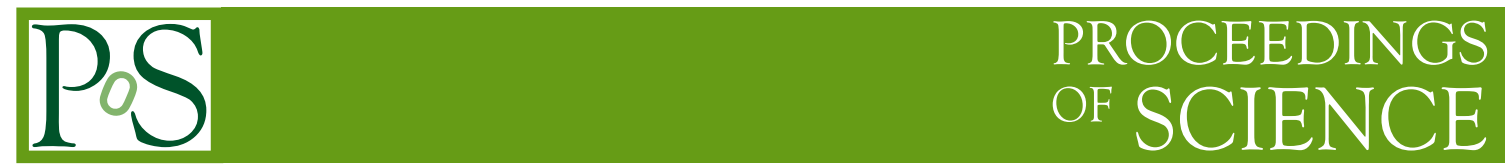

\title{
Hadrons in Nuclei
}

\author{
Laura Tolos* \\ Institut für Theoretische Physik, University of Frankfurt, Max-von-Laue-Str. 1, 60438 Frankfurt \\ am Main, Germany \\ Frankfurt Institute for Advanced Studies, University of Frankfurt, Ruth-Moufang-Str. 1, 60438 \\ Frankfurt am Main, Germany \\ Institute of Space Sciences (CSIC-IEEC), Campus Universitat Autònoma de Barcelona, Carrer \\ de Can Magrans, s/n, 08193 Cerdanyola del Vallès, Spain \\ E-mail: toloseth.physik.uni-frankfurt.de
}

A review on the state-of-the-art of heavy hadrons in nuclei is presented. In particular, the properties of mesons with strangeness and charm in matter are discussed, paying a special attention to the formation of exotic bound states in nuclei.

XVII International Conference on Hadron Spectroscopy and Structure - Hadron2017

25-29 September, 2017

University of Salamanca, Salamanca, Spain

${ }^{*}$ Speaker. 


\section{Introduction}

Over the last decades the properties of hadrons in nuclei have been matter of intense investigation. On the one hand, it is believed that hadrons in nuclei is an excellent scenario to test certain symmetries of the theory of the strong interaction, Quantum Chromodynamics (QCD), such as the chiral symmetry in the low-energy regime or heavy-quark symmetries as hadrons with charm or beauty content are produced in the laboratory. On the other hand, it is of crucial importance to understand the excitation mechanisms in the nucleus as well as the nature of certain excited hadronic states, whose structure could be studied in a hot and/or dense nuclear medium.

In order to address hadrons in nuclei one can resort to theoretical and/or experimental analyses $[1,2]$. From the theoretical side, there is an extensive variety of models that aim at understanding the properties of hadrons in nuclei, ranging from relativistic-mean field models (RMF), NambuJona-Lasinio schemes (NJL), quark-meson coupling models (QMC), QCD sum-rule studies to unitarized approaches based on effective theories or meson-exchange models. Experimentally, photon-, electron-, neutrino- and hadron-induced reactions as well as heavy-ion collisions (HiCs) offer a gateway to the properties of hadrons in nuclei. The measurement of transparency ratios is extremely useful for the analysis of the imaginary part of the hadron-nucleon interaction in matter, whereas the excitation functions and the meson-momentum distributions are of fundamental importance to understand the real part of the interaction [1]. Moreover, it is crucial to connect the theoretical predictions to the experimental results, using transport model calculations or collision models based on nuclear spectral functions, to fully understand the dynamics of hadrons in nuclei.

In this paper we concentrate on the analysis of the properties of mesons with strangeness and charm content that interact with nuclei. Nowadays, strange and charmed hadrons are being produced in nuclear and particle facilities, such as GSI, CERN or RHIC, while they are the subject of future experimental programs, such as FAIR, NICA or J-PARC.

\section{Strangeness in nuclei}

Strangeness in nuclei has received a lot attention in connection with the study of neutron stars interior [3], the properties of exotic atoms [4], and strangeness production in heavy-ion collisions (HICs) [5]. In particular, the dynamics of strange mesons, such as $\bar{K}$, in vacuum and in the nuclear medium is still a challenge for theory and experiments. In this section the $\bar{K} N$ interaction is studied, paying a special attention to the role of the $\Lambda(1405)$ and the formation of bound states, such as $\bar{K} N N$. Moreover, the production and propagation of strangeness in heavy-ion collisions (HICs) is investigated, in view of the present and forthcoming experimental programs on strangeness.

\section{1 $\bar{K} N$ interaction: the $\Lambda(1405)$}

The $\bar{K} N$ scattering in the $I=0$ channel is governed by the presence of the $\Lambda(1405)$, located only $27 \mathrm{MeV}$ below the $\bar{K} N$ threshold. The dynamical origin of the $\Lambda(1405)$ dates back more than 50 years ago to the work of Dalitz and Tuan [6]. Recently, it has been revisited by means of unitarized theories using meson-exchange models [7, 8] or chiral Lagrangians [9, 10, 11, 12, $13,14,15]$, these latter ones analyzing the effects of including a complete basis of meson-baryon channels, studying the differences in the regularization of the equations, including s- and u-channel 

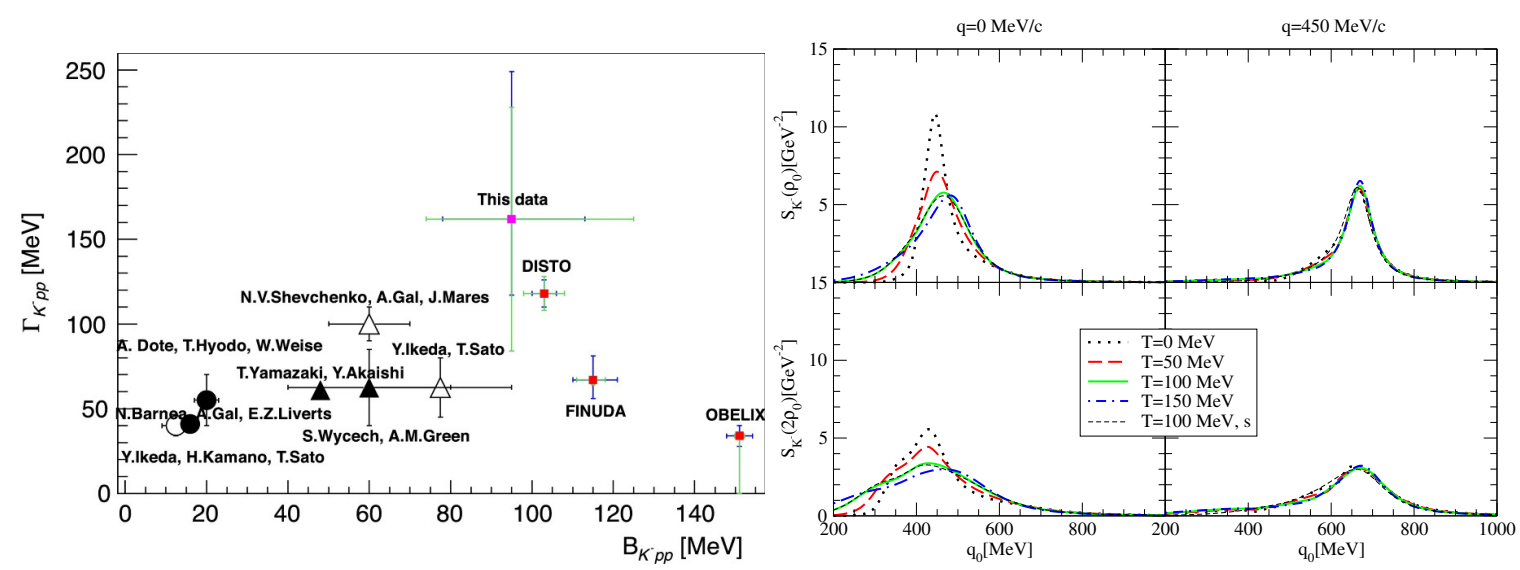

Figure 1: Left: Comparison of the binding energy and width of the $K^{-} p p$ between experiments and theoretical predictions, taken from [26]. Right: $\bar{K}$ spectral function for different densities, temperatures and momenta, taken from [27].

Born terms in the Lagrangian, implementing next-to-leading (NLO) contributions,... . All these recent efforts have culminated in establishing the $\Lambda(1405)$ as a superposition of two poles of the scattering matrix [16], that are generated dynamically from the unitarized coupled-channel scheme.

A renewed interest in the $\bar{K} N$ interaction has been developed in the past years after the availability of a more precise measurement of the energy shift and width of the $1 s$ state in kaonic hydrogen by the SIDDHARTA Collaboration at DAФNE [17], that has helped to clarify the discrepancies between the KEK [18, 19] and the DEAR [20,21] measurements. The obtained value of the energy shift is $\Delta E=283 \pm 36 \pm 6 \mathrm{eV}$ with a width of $\Gamma=541 \pm 89 \pm 22 \mathrm{eV}$, in good agreement with KEK results. Furthermore, the SIDDHARTA measurement has provided new constraints on the theoretical predictions reported in [22, 23, 24, 25].

\section{$2.2 \bar{K} N N$ state}

The dynamical generation of the two-pole structure of the $\Lambda(1405)$ indicates that the $\bar{K} N$ interaction might be attractive enough to produce bound states. Indeed, it has been suggested that $\bar{K}$-nuclear clusters may form, such as the $\bar{K} N N$ in isospin $I=1 / 2$. The $I=1 / 2 \bar{K} N N$ state has been extensively studied, both theoretically and experimentally, as shown in the left-hand side of Fig. 1 (see Ref. [26] and references therein). This state was initially seen by the FINUDA [28], DISTO [29] and OBELIX [30] Collaborations, but could be explained by means of conventional processes [31] or not be reproduced [32]. Experiments performed by the Spring8/LEPS [33], J-PARC E15 [34], AMADEUS [35] Collaborations do not find any state, or, if found [26, 36], may have other interpretation, such as as a possible $I=3 / 2, J^{\pi}=2^{+}$resonance near the $\pi \Sigma N$ threshold [37]. More recently, the J-PARC E15 experiment has found a structure near the $\bar{K} N N$ threshold [38], that has been interpreted as a $\bar{K} N N$ bound state with a binding energy of $\sim 20-40 \mathrm{MeV}$ [39].

\section{$2.3 \bar{K} N$ in matter}

Antikaonic atoms [4] give us information on the antikaon interaction with nucleons. The antikaon-nucleus potential has been extracted from best-fit analysis of antikaonic-atom data and 
some solutions agree with a very strongly attractive potential of the order of $-200 \mathrm{MeV}$ at normal saturation density $\rho_{0}$. However, some criticism has been raised because the antikaonic-atom data only tests matter at the surface of the nucleus. Recent analysis on $K^{-} N$ scattering amplitudes from chiral SU(3) effective field theories supplemented with phenomenological terms for $K^{-}$multinucleon interactions indicate that antikaonic atoms are insensitive to densities above $\rho_{0}$ [4].

Early works based on relativistic mean-field models [40] also obtained very deep potentials of a few hundreds of $\mathrm{MeVs}$ at $\rho_{0}$. However, later approaches on unitarized theories in coupled channels based on the chiral effective theory [41, 42] or on meson-exchange potentials [43, 44] obtain a potential much less attractive. In fact, in the unitarized coupled-channels models, the attraction is a consequence of the modified $s$-wave $\Lambda(1405)$ resonance in the medium due to Pauli blocking [45], together with the self-consistent inclusion of the $\bar{K}$ self-energy [41], and the implementation of self-energies of the mesons and baryons in the intermediate states [42]. As a result, the $\bar{K}$ spectral function can be obtained, as shown in the right-hand side of Fig. 1. The $\bar{K}$ spectral function shows that $\bar{K}$ in matter feel a slight attraction while acquiring a remarkable width. Moreover, the knowledge of higher-partial waves beyond $s$-wave [27, 46, 47, 48, 49] becomes essential for analyzing the results of $\mathrm{HiCs}$ at beam energies below $2 \mathrm{GeV}$ per nucleon [50, 51].

\subsection{Strangeness production in HICs}

The production of $K$ and $\bar{K}$ close to threshold has been extensively investigated in low-energy HICs by the KaoS [52], FOPI [53] and HADES Collaborations [54]. The analysis of experimental data together with microscopic transport approaches have permitted drawing several conclusions regarding the production mechanisms and the freeze-out conditions of strange mesons. However, the role of the in-medium properties of strange hadrons in their production and production in HiCs is still an open question. Recent results from HADES and FOPI indicate that, while the $K^{+}$shows a repulsive interaction in matter, the $\Phi$ decay into $K^{-}$washes out the effects of the $K^{-}$potential in the spectra and flow [55]. Therefore, more systematic and high statistic data on $K^{-}$production are necessary, while further information in elementary reactions is required.

\section{Open charm in nuclei}

The interest in the properties of open and hidden charmed mesons was triggered in $\mathrm{HiCs}$ due to the possible charmonium suppression as a probe for the formation of quark-gluon plasma (QGP). Nowadays, the nature of newly observed baryon and meson states with the charm degree of freedom is a matter of high interest in connection with many on-going experiments, such as BESIII, BelleII, ALICE, LHCb, amongst others, as well as with planned facilities, e.g. FAIR, NICA and the J-PARC upgrade. The goal is to understand whether these states can be accommodated within the quark model picture and/or qualify better as being dynamically generated via hadron-hadron scattering processes. To this end, a large part of the experimental program in hadronic physics at PANDA (FAIR) will be devoted to charmonium spectroscopy. Also, the CBM (FAIR) experiment will extend the GSI program for in-medium modification of hadrons in the light quark sector and provide the first insight into the charm-nucleus interaction. Indeed, the influence of medium modifications in the charmonium production at finite baryon densities would affect the formation of the QGP phase of QCD at high densities. 


\section{1 $D N$ interaction: the $\Lambda_{c}(2595)$}

Given the success of unitarized coupled-channel approaches in the description of some of the existing experimental data in the light-quark sector, the charm degree of freedom has been recently incorporated in these models and several experimental states have been described as dynamicallygenerated baryon molecules (see Ref. [56] and references therein). This is the case, for example, of the $\Lambda_{c}(2595)$, which is the charmed counterpart of the $\Lambda(1405)$.

Whereas a separable potential for the bare meson-baryon interaction with no strange degree of freedom was assumed in [57], later on unitarized approaches were based on a bare meson-baryon interaction saturated with the $t$-channel exchange of vector mesons between pseudoscalar mesons and baryons in the zero-range approximation [58, 59] or using the full $t$-dependence [60]. Other approaches have made use of the Jülich meson-exchange model $[8,61]$, while some others have relied on the hidden gauge formalism [62, 63]. More recent schemes incorporate heavy-quark symmetry constraints explicitly, such as those based on a pion-exchange model $[64,65]$ or on an extended Weinberg-Tomozawa interaction for four flavors that includes pseudoscalar and vector mesons together with $1 / 2^{+}$and $3 / 2^{+}$baryons $[66,67,68,69]$. In all these unitarized coupledchannel models, the $\Lambda_{c}(2595)$ is obtained dynamically, some of them $[8,58,66,67,68,69]$ even obtaining a double-pole structure, in a similar manner as found for the $\Lambda(1405)$.

\section{2 $\bar{D} N$ interaction}

The $C=-1$ has also been investigated within unitarized coupled-channel models, pion-exchange schemes with heavy-quark symmetry constraints or chiral quark models [58, 61, 64, 65, 67, 70]. Interestingly, some of the models find a $J=1 / 2$ state close to the $\bar{D} N$ threshold [64, 65, 67]. In Ref. [67] this state was generated by the $\bar{D} N$ and $\bar{D}^{*} N$ coupled channel dynamics, and it appears to be a consequence of treating heavy pseudoscalars and heavy vector mesons on an equal footing, because no resonance would be generated unless $\bar{D}^{*} N$ channel is considered.

\section{3 $D N N$ and $\bar{D} N N$ states}

Given the fact that the $D N$ and $\bar{D} N$ interactions are so attractive that allow for the formation of bound states, the question arises whether $D$ or $\bar{D}$-nuclear clusters may form. In [71] a $I=1 / 2$, $J=0^{-} D N N$ state was found with mass $3500 \mathrm{MeV}$ and width $\sim 20-40 \mathrm{MeV}$, being interpreted as a quasibound state of the $\Lambda_{c}(2595)$ and a nucleon. Moreover, in [72] a state with $I=1 / 2$, $J=0^{-}$and $5.2 \mathrm{MeV}$ binding was found together with a $I=1 / 2, J=1^{-}$state at $111.2 \mathrm{MeV}$ above threshold.

\subsection{Open charm in matter}

The properties of open-charm mesons in matter have been object of theoretical interest due to the consequences for charmonium suppression, as observed at SPS energies by the NA50 collaboration. The change of the properties of D mesons in matter would modify the $J / \Psi$ absorption in a hot and dense nuclear medium and can provide an explanation for $J / \Psi$ suppression. Furthermore, there have been speculations about the existence of $D$-meson bound states in nuclei [75].

Several theoretical works have addressed the properties of open-charm mesons in dense baryonic matter: QMC schemes [75], QCD sum-rule approaches [76, 77, 78], NJL models [79], chiral 


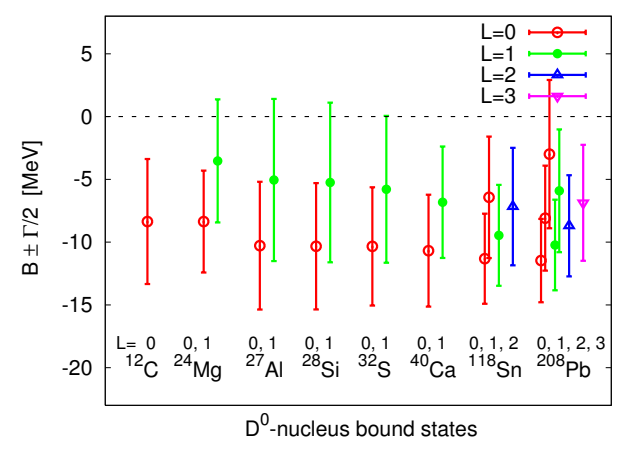

Figure 2: $D^{0}$-nucleus bound states, taken from [73].
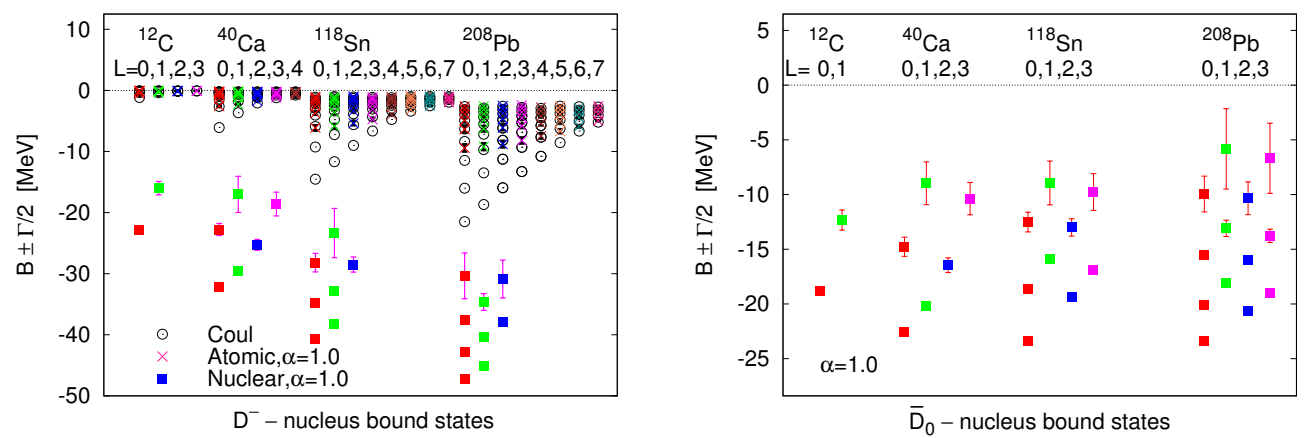

Figure 3: $D^{-}$and $\bar{D}^{0}$ - nucleus bound states, taken from [74].

effective models in hot and dense matter [80] or pion-exchange approaches with heavy-quark symmetry constraints [81]. The full spectral features (mass and width) of the open-charm mesons in dense nuclear matter have been obtained in self-consistent unitarized coupled-channel schemes, where the intermediate meson-baryon propagators contain different sources of density dependence $[74,57,59,82,83,84,85,86]$.

\subsection{D-mesic nuclei}

A possible experimental scenario for the detection of the changes in matter of the properties of open-charm mesons would be the formation of D-mesic nuclei, where a D-meson binds in nuclear orbits. In fact, $D$ and $\bar{D}$-meson bound states in ${ }^{208} \mathrm{~Pb}$ were predicted in Ref. [75], relying upon an attractive $D$ and $\bar{D}$-meson potential in the nuclear medium, obtained within a QMC model.

Within the unitarized coupled-channel model of Ref. [73], it is found that $D^{0}$-nucleus states are weakly bound (see Fig. 2), in contrast to previous results using the QMC model [75], while having significant widths. The best chances for observation of bound states are in the region of ${ }^{24} \mathrm{Mg}$, provided an orbital angular momentum separation can be done, where there is only one $s-$ bound state and its half width is about a factor of two smaller than the binding energy (see Fig. 2). With regards to $D^{+}$-nuclear states, the Coulomb interaction prevents the formation of observable bound states. As for $\bar{D}$-mesic nuclei, not only $D^{-}$but also $\bar{D}^{0}$ bind in nuclei as seen in Fig. 3. The 
spectrum contains states of atomic and of nuclear types for all nuclei for $D^{-}$while, as expected, only nuclear states are present for $\bar{D}^{0}$ in nuclei. Compared to the pure Coulomb levels, the atomic states are less bound. The nuclear ones are more bound and may present a sizable width, existing only for low angular momenta [74]. This is in contrast to [75] for ${ }^{208} \mathrm{~Pb}$, but close to results in [81].

The experimental detection of $D$ and $\bar{D}$-meson bound states is, though, a difficult task [87]. The formation of D-mesic nuclei with antiprotons beams at PANDA (FAIR) might be possible if $(\bar{p}, \mathrm{D}+\mathrm{N})$ or $(\bar{p}, \mathrm{D}+2 \mathrm{~N})$ reactions with small or even zero momentum transfer are produced, although the formation cross sections could be suppressed because of the complexity of the reaction mechanisms. Other competing mechanisms could involve the emission of pions by intermediate $D^{*}$ or $\bar{D}^{*}$ with subsequent trapping of the pseudoscalar charmed mesons by the final nucleus [87].

\section{Acknowledgements}

L.T. acknowledges support from the Heisenberg Programme of the Deutsche Forschungsgemeinschaft under the Project Nr. 383452331, the Ramón y Cajal research programme, FPA201343425-P and FPA2016-81114-P Grants from MINECO, and THOR COST Action CA15213.

\section{References}

[1] V. Metag, M. Nanova and E. Ya. Paryev, Meson-nucleus potentials and the search for meson-nucleus bound states, Prog. Part. Nucl. Phys. 97 (2017) 199-260, [1706.09654].

[2] R. Rapp et al., In-medium excitations, Lect. Notes Phys. 814 (2011) 335-529.

[3] A. L. Watts et al., Colloquium : Measuring the neutron star equation of state using x-ray timing, Rev. Mod. Phys. 88 (2016) 021001, [1602.01081].

[4] E. Friedman and A. Gal, $K^{-} N$ amplitudes below threshold constrained by multinucleon absorption, Nucl. Phys. A959 (2017) 66-82, [1610.04004].

[5] C. Hartnack, H. Oeschler, Y. Leifels, E. L. Bratkovskaya and J. Aichelin, Strangeness Production close to Threshold in Proton-Nucleus and Heavy-Ion Collisions, Phys. Rept. 510 (2012) 119-200, [1106.2083].

[6] R. H. Dalitz and S. F. Tuan, A possible resonant state in pion-hyperon scattering, Phys. Rev. Lett. 2 (1959) 425-428.

[7] A. Mueller-Groeling, K. Holinde and J. Speth, $K$ - $N$ interaction in the meson exchange framework, Nucl. Phys. A513 (1990) 557-583.

[8] J. Haidenbauer, G. Krein, U.-G. Meissner and L. Tolos, DN interaction from meson exchange, Eur. Phys. J. A47 (2011) 18, [1008.3794].

[9] N. Kaiser, P. B. Siegel and W. Weise, Chiral dynamics and the low-energy kaon - nucleon interaction, Nucl. Phys. A594 (1995) 325-345, [nucl-th/ 9505043 ].

[10] E. Oset and A. Ramos, Nonperturbative chiral approach to s wave anti-K N interactions, Nucl. Phys. A635 (1998) 99-120, [nucl-th/9711022].

[11] J. A. Oller and U. G. Meissner, Chiral dynamics in the presence of bound states: Kaon nucleon interactions revisited, Phys. Lett. B500 (2001) 263-272, [hep-ph/ 0011146 ]. 
[12] M. F. M. Lutz and E. E. Kolomeitsev, Relativistic chiral SU(3) symmetry, large $N(c)$ sum rules and meson baryon scattering, Nucl. Phys. A700 (2002) 193-308, [nucl-th/0105042].

[13] C. Garcia-Recio, J. Nieves, E. Ruiz Arriola and M. J. Vicente Vacas, $S=-1$ meson baryon unitarized coupled channel chiral perturbation theory and the S(01) Lambda(1405) and Lambda(1670) resonances, Phys. Rev. D67 (2003) 076009, [hep-ph/ 0210311$].$

[14] B. Borasoy, R. Nissler and W. Weise, Chiral dynamics of kaon-nucleon interactions, revisited, Eur. Phys. J. A25 (2005) 79-96, [hep-ph/0505239].

[15] J. A. Oller, On the strangeness -1 S-wave meson-baryon scattering, Eur. Phys. J. A28 (2006) 63-82, [hep-ph/0603134].

[16] D. Jido, J. A. Oller, E. Oset, A. Ramos and U. G. Meissner, Chiral dynamics of the two Lambda(1405) states, Nucl. Phys. A725 (2003) 181-200, [nucl-th/0303062].

[17] SIDDHARTA collaboration, M. Bazzi et al., A New Measurement of Kaonic Hydrogen X-rays, Phys. Lett. B704 (2011) 113-117, [1105.3090].

[18] M. Iwasaki et al., Observation of the kaonic hydrogen K(alpha) x-ray, Phys. Rev. Lett. 78 (1997) 3067-3069.

[19] T. M. Ito et al., Observation of kaonic hydrogen atom x rays, Phys. Rev. C58 (1998) 2366-2382.

[20] DEAR collaboration, G. Beer et al., Measurement of the kaonic hydrogen X-ray spectrum, Phys. Rev. Lett. 94 (2005) 212302.

[21] DEAR collaboration, M. Cargnelli et al., Kaonic hydrogen measurement with DEAR at DAPHNE, Int. J. Mod. Phys. A20 (2005) 341-348.

[22] Y. Ikeda, T. Hyodo and W. Weise, Improved constraints on chiral SU(3) dynamics from kaonic hydrogen, Phys. Lett. B706 (2011) 63-67, [1109.3005].

[23] Z.-H. Guo and J. A. Oller, Meson-baryon reactions with strangeness -1 within a chiral framework, Phys. Rev. C87 (2013) 035202, [1210.3485].

[24] M. Mai and U.-G. Meissner, New insights into antikaon-nucleon scattering and the structure of the Lambda(1405), Nucl. Phys. A900 (2013) 51 - 64, [1202.2030].

[25] A. Feijoo, V. K. Magas and A. Ramos, The $\bar{K} N \rightarrow K \Xi$ reaction in coupled channel chiral models up to next-to-leading order, Phys. Rev. C92 (2015) 015206, [1502.07956].

[26] T. Nagae, Production of the $K^{-}$pp-like structure in the $d\left({ }^{+}, K^{+}\right)$reaction at $1.69 \mathrm{GeV} / \mathrm{c}$, Nucl. Phys. A954 (2016) 94-113.

[27] L. Tolos, D. Cabrera and A. Ramos, Strange mesons in nuclear matter at finite temperature, Phys. Rev. C78 (2008) 045205, [0 807 . 2947].

[28] FINUDA collaboration, M. Agnello et al., Evidence for a kaon-bound state $K$ - p p produced in Kabsorption reactions at rest, Phys. Rev. Lett. 94 (2005) 212303.

[29] T. Yamazaki et al., Indication of a deeply bound compact K-pp state formed in the pp -> p Lambda K+ reaction at 2.85 GeV, Phys. Rev. Lett. 104 (2010) 132502, [1 002 . 352 6].

[30] G. Bendiscioli, T. Bressani, L. Lavezzi and P. Salvini, A new analysis improving the evidence of a narrow peak in the invariant-mass distribution of the Lambda p system observed in the anti-p annihilation at rest on He-4, Eur. Phys. J. A40 (2009) 11-22. 
[31] A. Ramos, V. K. Magas, E. Oset and H. Toki, The antikaon-nucleus interaction and alternative views to deeply bound antikaonic nuclear systems, Nucl. Phys. A804 (2008) 219-230.

[32] HADES collaboration, G. Agakishiev et al., Partial Wave Analysis of the Reaction $p(3.5 \mathrm{GeV})+p \rightarrow p K^{+} \Lambda$ to Search for the "ppK $\mathrm{K}^{-" B o u n d}$ State, Phys. Lett. B742 (2015) 242-248, [1410.8188].

[33] LEPS collaboration, A. O. Tokiyasu et al., Search for $K^{-}$pp bound state via $\gamma d \rightarrow K^{+} \pi^{-} X$ reaction at $E_{\gamma}=1.5-2.4 \mathrm{GeV}$, Phys. Lett. B728 (2014) 616-621, [1306.5320].

[34] J-PARC E15 collaboration, T. Hashimoto et al., Search for the deeply bound $K^{-}$pp state from the semi-inclusive forward-neutron spectrum in the in-flight $K^{-}$reaction on helium-3, PTEP 2015 (2015) 061D01, [1408.5637].

[35] O. Vázquez Doce et al., $K^{-}$absorption on two nucleons and $p p K^{-}$bound state search in the $\Sigma^{0} p$ final state, Phys. Lett. B758 (2016) 134-139, [1511. 04496$].$

[36] Y. Ichikawa et al., Observation of the " $K^{-} p p^{\prime \prime-l i k e ~ s t r u c t u r e ~ i n ~ t h e ~} d\left(\pi^{+}, K^{+}\right)$reaction at $1.69 \mathrm{GeV} / \mathrm{c}$, PTEP 2015 (2015) 021D01, [1411.6708].

[37] H. Garcilazo and A. Gal, Relativistic three-body calculations of a $Y=1, I=3 / 2$, $J P=2+\pi \Lambda N-\pi \Sigma N$ dibaryon, Nucl. Phys. A897 (2013) 167-178, [1209.3992].

[38] J-PARC E15 collaboration, Y. Sada et al., Structure near $K^{-}+p+p$ threshold in the in-flight ${ }^{3} \mathrm{He}\left(K^{-}, \Lambda p\right) n$ reaction, PTEP 2016 (2016) 051D01, [1601.06876].

[39] T. Sekihara, E. Oset and A. Ramos, On the structure observed in the in-flight ${ }^{3} \mathrm{He}\left(\mathrm{K}^{-}, \Lambda \mathrm{p}\right) n$ reaction at J-PARC, PTEP 2016 (2016) 123D03, [1607. 020 58].

[40] J. Schaffner, J. Bondorf and I. N. Mishustin, In-medium production of kaons at the mean field level, Nucl. Phys. A625 (1997) 325-346, [nucl-th/9607058].

[41] M. Lutz, Nuclear kaon dynamics, Phys. Lett. B426 (1998) 12-20, [nucl-th/9709073].

[42] A. Ramos and E. Oset, The Properties of anti-K in the nuclear medium, Nucl. Phys. A671 (2000) 481-502, [nucl-th/9906016].

[43] L. Tolos, A. Ramos, A. Polls and T. T. S. Kuo, Partial wave contributions to the anti-kaon potential at finite momentum, Nucl. Phys. A690 (2001) 547-566, [nucl-th/ 0007042 ].

[44] L. Tolos, A. Ramos and A. Polls, The Anti-kaon nuclear potential in hot and dense matter, Phys. Rev. C65 (2002) 054907, [nucl-th/0202057].

[45] V. Koch, K- - proton scattering and the Lambda (1405) in dense matter, Phys. Lett. B337 (1994) 7-13, [nucl-th/9406030].

[46] L. Tolos, A. Ramos and E. Oset, Chiral approach to antikaon s and p-wave interactions in dense nuclear matter, Phys. Rev. C74 (2006) 015203, [nucl-th/ 0603033 ].

[47] M. F. M. Lutz, C. L. Korpa and M. Moller, Antikaons and hyperons in nuclear matter with saturation, Nucl. Phys. A808 (2008) 124-159, [0707.1283].

[48] D. Cabrera, A. Polls, A. Ramos and L. Tolos, Energy weighted sum rules for mesons in hot and dense matter, Phys. Rev. C80 (2009) 045201, [0 903 . 1171].

[49] D. Cabrera, L. Tolós, J. Aichelin and E. Bratkovskaya, Antistrange meson-baryon interaction in hot and dense nuclear matter, Phys. Rev. C90 (2014) 055207, [1406.2570]. 
[50] W. Cassing, L. Tolos, E. L. Bratkovskaya and A. Ramos, Anti-kaon production in A+A collisions at SIS energies within an off-shell G matrix approach, Nucl. Phys. A727 (2003) 59-94, [nucl-th/0304006].

[51] L. Tolos, A. Polls, A. Ramos and J. Schaffner-Bielich, $K-/ K+$ ratio in heavy ion collisions at GSI with an antikaon selfenergy in hot and dense matter, Phys. Rev. C68 (2003) 024903, [nucl-th/0302082].

[52] A. Forster et al., Production of K+ and of K-Mesons in Heavy-Ion Collisions from 0.6 to 2.0-A-GeV Incident Energy, Phys. Rev. C75 (2007) 024906, [nucl-ex/ 0701014 ].

[53] FOPI collaboration, X. Lopez et al., Measurement of $K^{*}(892)^{0}$ and $K^{0}$ mesons in Al+Al collisions at 1.9A GeV, Phys. Rev. C81 (2010) 061902, [1006.1905].

[54] HADES collaboration, G. Agakishiev et al., Medium effects in proton-induced $K^{0}$ production at 3.5 GeV, Phys. Rev. C90 (2014) 054906, [1404.7011].

[55] Y. Leifels, plenary talk at SQM2017 conference, .

[56] L. Tolos, Charming mesons with baryons and nuclei, Int. J. Mod. Phys. E22 (2013) 1330027, [1309.7305].

[57] L. Tolos, J. Schaffner-Bielich and A. Mishra, Properties of D-mesons in nuclear matter within a self-consistent coupled-channel approach, Phys. Rev. C70 (2004) 025203, [nucl-th/ 0404064 ].

[58] J. Hofmann and M. F. M. Lutz, Coupled-channel study of crypto-exotic baryons with charm, Nucl. Phys. A763 (2005) 90-139, [hep-ph/0507071].

[59] T. Mizutani and A. Ramos, D mesons in nuclear matter: A DN coupled-channel equations approach, Phys. Rev. C74 (2006) 065201, [hep-ph/ 0607257$].$

[60] C. E. Jimenez-Tejero, A. Ramos and I. Vidana, Dynamically generated open charmed baryons beyond the zero range approximation, Phys. Rev. C80 (2009) 055206, [0 907 . 5316].

[61] J. Haidenbauer, G. Krein, U.-G. Meissner and A. Sibirtsev, Anti-D N interaction from meson-exchange and quark-gluon dynamics, Eur. Phys. J. A33 (2007) 107-117, [0 704 . 3668].

[62] J.-J. Wu, R. Molina, E. Oset and B. S. Zou, Prediction of narrow $N^{*}$ and $\Lambda^{*}$ resonances with hidden charm above 4 GeV, Phys. Rev. Lett. 105 (2010) 232001, [1007. 0573].

[63] E. Oset, A. Ramos, E. J. Garzon, R. Molina, L. Tolos, C. W. Xiao et al., Interaction of vector mesons with baryons and nuclei, Int. J. Mod. Phys. E21 (2012) 1230011, [1210.3738].

[64] Y. Yamaguchi, S. Ohkoda, S. Yasui and A. Hosaka, Hadronic molecules for charmed and bottom baryons near thresholds, Phys. Rev. D87 (2013) 074019, [1301.4557].

[65] A. Hosaka, T. Hyodo, K. Sudoh, Y. Yamaguchi and S. Yasui, Heavy Hadrons in Nuclear Matter, Prog. Part. Nucl. Phys. 96 (2017) 88-153, [1606.08685].

[66] C. Garcia-Recio, V. K. Magas, T. Mizutani, J. Nieves, A. Ramos, L. L. Salcedo et al., The s-wave charmed baryon resonances from a coupled-channel approach with heavy quark symmetry, Phys. Rev. D79 (2009) 054004, [0 807 .2969].

[67] D. Gamermann, C. Garcia-Recio, J. Nieves, L. L. Salcedo and L. Tolos, Exotic dynamically generated baryons with negative charm quantum number, Phys. Rev. D81 (2010) 094016, [1002 . 2763].

[68] O. Romanets, L. Tolos, C. Garcia-Recio, J. Nieves, L. L. Salcedo and R. G. E. Timmermans, Charmed and strange baryon resonances with heavy-quark spin symmetry, Phys. Rev. D85 (2012) 114032, [1202.2239]. 
[69] C. Garcia-Recio, J. Nieves, O. Romanets, L. L. Salcedo and L. Tolos, Hidden charm N and Delta resonances with heavy-quark symmetry, Phys. Rev. D87 (2013) 074034, [1302 . 6938].

[70] T. F. Carames and A. Valcarce, ND̄ system: A challenge for $\overline{\mathrm{P}} A N D A$, Phys. Rev. D85 (2012) 094017, [1204.5502].

[71] M. Bayar, C. W. Xiao, T. Hyodo, A. Dote, M. Oka and E. Oset, Energy and width of a narrow I=1/2 DNN quasibound state, Phys. Rev. C86 (2012) 044004, [12 05 . 2275].

[72] Y. Yamaguchi, S. Yasui and A. Hosaka, Exotic dibaryons with a heavy antiquark, Nucl. Phys. A927 (2014) 110-118, [1309.4324].

[73] C. Garcia-Recio, J. Nieves and L. Tolos, D mesic nuclei, Phys. Lett. B690 (2010) 369-375, [1004.2634].

[74] C. Garcia-Recio, J. Nieves, L. L. Salcedo and L. Tolos, $D^{-}$mesic atoms, Phys. Rev. C85 (2012) 025203, [1111.6535].

[75] K. Tsushima, D.-H. Lu, A. W. Thomas, K. Saito and R. H. Landau, Charmed mesic nuclei, Phys. Rev. C59 (1999) 2824-2828, [nucl-th/9810016].

[76] A. Hayashigaki, Mass modification of D meson at finite density in QCD sum rule, Phys. Lett. B487 (2000) 96-103, [nucl-th/0001051].

[77] T. Hilger, B. Kampfer and S. Leupold, Chiral QCD sum rules for open charm mesons, Phys. Rev. C84 (2011) 045202, [1109.1229].

[78] K. Suzuki, P. Gubler and M. Oka, D meson mass increase by restoration of chiral symmetry in nuclear matter, Phys. Rev. C93 (2016) 045209, [1511. 04513 ].

[79] D. Blaschke, P. Costa and Yu. L. Kalinovsky, D mesons at finite temperature and density in the PNJL model, Phys. Rev. D85 (2012) 034005, [1107.2913].

[80] A. Mishra, E. L. Bratkovskaya, J. Schaffner-Bielich, S. Schramm and H. Stoecker, Mass modification of D meson in hot hadronic matter, Phys. Rev. C69 (2004) 015202, [nucl-th/0308082].

[81] S. Yasui and K. Sudoh, $\bar{D}$ and B mesons in nuclear medium, Phys. Rev. C87 (2013) 015202, [1207.3134].

[82] L. Tolos, J. Schaffner-Bielich and H. Stoecker, D-mesons: In-medium effects at FAIR, Phys. Lett. B635 (2006) 85-92, [nucl-th/ 0509054 ].

[83] M. F. M. Lutz and C. L. Korpa, Open-charm systems in cold nuclear matter, Phys. Lett. B633 (2006) 43-48, [nucl-th/0510006].

[84] L. Tolos, A. Ramos and T. Mizutani, Open charm in nuclear matter at finite temperature, Phys. Rev. C77 (2008) 015207, [0710.2684].

[85] C. E. Jimenez-Tejero, A. Ramos, L. Tolos and I. Vidana, Open charm meson in nuclear matter at finite temperature beyond the zero range approximation, Phys. Rev. C84 (2011) 015208, [1102 . 4786 ].

[86] L. Tolos, C. Garcia-Recio and J. Nieves, The Properties of $D$ and $D^{*}$ mesons in the nuclear medium, Phys. Rev. C80 (2009) 065202, [0905.4859].

[87] J. Yamagata-Sekihara, C. Garcia-Recio, J. Nieves, L. L. Salcedo and L. Tolos, Formation spectra of charmed meson-nucleus systems using an antiproton beam, Phys. Lett. B754 (2016) 26-32, [1512.03240]. 